\title{
Channel Reconstruction Based Multiuser Precoding with Limited Feedback
}

\author{
Mert Özateş \\ EEE Dept., Bilkent University \\ Ankara, Turkey \\ ozates@ee.bilkent.edu.tr
}

\author{
Mohammad Kazemi \\ EEE Dept., Bilkent University \\ Ankara, Turkey \\ kazemi@ee.bilkent.edu.tr
}

\author{
Çağrı Göken \\ ASELSAN A.Ş. \\ Ankara, Turkey \\ cgoken@aselsan.com.tr
}

\author{
Tolga M. Duman \\ EEE Dept., Bilkent University \\ Ankara, Turkey \\ duman@ee.bilkent.edu.tr
}

\begin{abstract}
We consider the downlink of a multiuser multipleinput multiple-output (MU-MIMO) system, where each user feeds back a partial channel state information (CSI), namely, the quantized version of the dominant eigenvector of its channel covariance matrix, to the base station (BS) for precoding. Specifically, we propose a downlink multiuser precoding scheme by first reconstructing the equivalent channel matrix of each user via a limited feedback, and then by employing a precoder to suppress the multiuser interference at the receivers. For the single stream case, a signal-to-leakage-and-noise ratio (SLNR) based precoding is employed, while for the full stream case with limited feedback, we employ a lattice reduction aided block diagonalization type precoding with suitable modifications at the receiver side. Extensive numerical examples which are provided using the 5G new radio (5G-NR) channel models demonstrate that the proposed schemes outperform the existing eigenvector based algorithms, and they are more robust against the downlink channel estimation errors.
\end{abstract}

Index Terms-Multiuser MIMO, limited feedback, channel reconstruction, SLNR, lattice reduction, 5G-NR.

\section{INTRODUCTION}

Knowledge of the channel state information (CSI) at a base station (BS) plays an important role in achieving higher throughputs in future cellular networks. In order for the BS to obtain the downlink CSI, the users need to feed back their downlink channel matrix (e.g., estimated through downlink pilot training). This induces a huge amount of overhead to the system, which in turn decreases the efficacy of CSI utilization. To resolve this issue, limited feedback techniques are employed for which either a quantized version of the channel coefficients (or, a property of channel matrix such as the dominant eigenvector) or their corresponding indices from a predefined codebook are fed back to the BS [1]. The BS then utilizes this information to precode the data for downlink transmission.

In recent years, signal-to-leakage-and-noise ratio (SLNR) metric has been widely utilized for precoder design where the leakage is defined as the interference caused by the precoding vector of each user at the other users' receivers [7]. There are also a few works that investigate the SLNR based precoding

This research is partially supported by ASELSAN Inc. under the "5G Platform" project which is partly funded by the Scientific and Technological Research Council of Turkey (TUBITAK) under the project number 1160206.

Mert Ozates' work is partially supported by Vodafone within the framework of 5G and Beyond Joint Graduate Support Programme coordinated by Information and Communication Technologies Authority. with limited feedback in cellular networks [2]-[5]. In [3], the authors design a modified version of the Grassmannian codebook for feeding back the channel coefficients in a multiuser multiple-input multiple-output (MU-MIMO) system with single antenna users. They show that, when combined with the SLNR precoding, the new codebook outperforms the conventional ones such as random vector quantization (RVQ). Utilizing the lattice reduction (LR) technique [9] and employing a minimum mean square error (MMSE) channel inversion, the authors in [11] introduce an LR-aided block diagonalization (BD) type precoding algorithm for the full stream transmission in multiuser MIMO systems. This algorithm, which builds upon the generalized MMSE channel inversion (GMI) precoding algorithm [10], is called the LR-aided simplified GMI (LR-SGMI). In [2], the multiple-antenna users feed back the elements of the upper triangular matrix after a QR decomposition of the channel, and the quantization levels are designed based on the statistical distribution of the elements of the channel matrix. It is shown that this approach reduces the feedback overhead while achieving almost the same performance as feeding back the channel coefficients themselves.

In this paper, we assume that the multi-antenna users feed back the dominant eigenvector (i.e., the eigenvector corresponding to the largest eigenvalue) of the covariance matrix of their channel to the BS using a Type-2 codebook in 5G New Radio (5G-NR), and propose an algorithm to reconstruct the equivalent channel matrices at the BS, which are used in downlink precoding. We employ an SLNR maximizing precoder for the single stream case. Also, for the full stream case, we employ the LR-S-GMI algorithm with an inner MMSE precoder, called LR-S-GMI-MMSE. We show that with some suitable modifications at the receiver side, LR-S-GMI-MMSE becomes highly promising for downlink precoding with limited feedback.

There are multiuser precoding methods such as those based on SLNR maximization, which assume the channel matrix knowledge at the transmitter explicitly. There are also alternative methods such as the singular value distribution based signature matrix inversion (SVD-SI) [12] and the successive MMSE (S-MMSE) based method proposed in [13] which process and transform the CSI feedback to obtain updated precoding matrices, which can handle the multiuser interference. The newly proposed algorithm in this paper brings a new 
perspective to precoding in MU-MIMO systems, where the CSI feedback is first used to obtain an equivalent channel matrix instead of directly being used for the precoder calculation. Once the approximate channel matrix is obtained, existing precoding methods such as SLNR based or LR-S-GMI-MMSE (along with a modified receiver) can be applied to obtain the precoders. In other words, the proposed algorithm decouples the precoder selection task under limited feedback into two independent blocks, namely, channel reconstruction with limited feedback and precoder selection based on the equivalent channel matrix. Hence, it is possible to generate different versions of the proposed algorithm with our approach, bringing additional flexibility and ensuring an efficient operation in terms of the tradeoff between the feedback overhead and performance. Finally, we note that even though the proposed algorithm is tested using 5G Type-2 codebook, it can be used for other legacy or independent wireless communication systems involving a certain form of CSI feedback.

The remainder of the paper is organized as follows. The system model and a brief explanation of the limited feedback process is presented in Section II. In Section III, the proposed limited feedback precoding schemes are explained. The numerical results are presented in Section IV. Finally, the paper is concluded in Section V.

\section{System Model AND PREliminaries}

\section{A. System Model}

We consider a wideband multiuser MIMO system where a BS with $N_{t}$ antennas serves $K$ users each with $N_{r}$ antennas. We assume that the users have perfect knowledge of their downlink channel (e.g., through a downlink pilot training). The users feed back a partial CSI for each subband, namely, the dominant eigenvector of the covariance matrix of its downlink channel to the BS. However, since feeding back the actual dominant eigenvector is costly, each user first quantizes it using a discrete Fourier transform (DFT) Type-2 codebook in 5GNR [6], and then feeds it back to the BS. Finally, having the quantized eigenvectors of the covariance matrices of the downlink channels, the BS sends data symbols to the users by utilizing some precoding techniques.

The received signal $\mathbf{y}_{k, j} \in \mathcal{C}^{N_{r}}$ at the $k$-th user in the $j$-th subcarrier is given by

$$
\mathbf{y}_{k, j}=\mathbf{H}_{k, j} \mathbf{P}_{k} \mathbf{s}_{k, j}+\mathbf{H}_{k, j} \sum_{i=1, i \neq k}^{K} \mathbf{P}_{k} \mathbf{s}_{i, j}+\mathbf{n}_{k, j},
$$

where $\mathbf{H}_{k, j} \in \mathcal{C}^{N_{r} \times N_{t}}$ is the channel matrix of the $k$-th user in the $j$-th subcarrier, $\mathbf{n}_{k, j} \in \mathcal{C}^{N_{r}}$ is the circularly symmetric complex Gaussian noise at the $k$-th user in the $j$-th subcarrier with independent and identically distributed (i.i.d.) entries of zero mean and variance $\sigma_{n}^{2}, \mathbf{P}_{k} \in \mathcal{C}^{N_{t} \times r_{k}}$ is the precoding matrix of the $k$-th user, and $\mathbf{s}_{k, j} \in \mathcal{C}^{r_{k}}$ is the desired data vector of the $k$-th user in the $j$-th subcarrier where $r_{k}$ is the number of data streams for the $k$-th user.

In the following, we explain how the quantized CSI feedback to represent the dominant eigenvectors of the downlink channel can be obtained using DFT Type-2 codebook in 5G-NR.

\section{B. DFT Type-2 Structure in 5G-NR}

In 5G-NR, two types of codebooks are defined for CSI feedback, namely, Type-1 and Type-2. In both of them, DFT beams, which are constructed from an oversampled DFT matrix, are used, but only one beam per stream is employed in Type-1 to represent the channel. In order to obtain a more accurate representation, Type-2 codebook is utilized for MU-MIMO operation [6].

In Type-2 codebooks, $L$ beams (up to 4) are allowed to represent the channel, which increases the resolution of the fed-back CSI. The multiple beams are linearly combined with respective amplitudes and phases chosen from a finite set of values to construct the DFT Type- 2 codebook elements, which are used to estimate the dominant eigenvector of the covariance matrix to be fed back to the BS.

Assuming a two-beam representation $(L=2)$, for simplicity, a DFT Type-2 vector $\mathbf{w}$ can be written as follows:

$$
\begin{aligned}
\mathbf{w} & =\left[\mathbf{x}_{0}^{T}, \mathbf{x}_{1}^{T}\right]^{T}, \\
\mathbf{x}_{0} & =\mathbf{b}^{(0)} \rho_{0}^{(0)} \varphi_{0}^{(0)}+\mathbf{b}^{(1)} \rho_{0}^{(1)} \varphi_{0}^{(1)}, \\
\mathbf{x}_{1} & =\mathbf{b}^{(0)} \rho_{1}^{(0)} \varphi_{1}^{(0)}+\mathbf{b}^{(1)} \rho_{1}^{(1)} \varphi_{1}^{(1)},
\end{aligned}
$$

where $\mathbf{b}^{(0)}$ and $\mathbf{b}^{(1)}$ are the DFT vectors orthogonal to each other, and $\rho_{i}^{(j)}$ 's and $\varphi_{i}^{(j)}$ 's are the amplitude and phase coefficients, respectively. Vector $\mathbf{w}$ can also be written as a product of a matrix and a vector as

$$
\begin{aligned}
& \mathbf{w}=\mathbf{W}_{\mathbf{1}} \mathbf{w}_{\mathbf{2}}, \\
& \mathbf{W}_{\mathbf{1}}=\left[\begin{array}{cccc}
\mathbf{b}^{(0)} & \mathbf{b}^{(1)} & 0 & 0 \\
0 & 0 & \mathbf{b}^{(0)} & \mathbf{b}^{(1)}
\end{array}\right], \mathbf{w}_{\mathbf{2}}=\left[\begin{array}{c}
\rho_{0}^{(0)} \varphi_{0}^{(0)} \\
\rho_{0}^{(1)} \varphi_{0}^{(1)} \\
\rho_{1}^{(0)} \varphi_{1}^{(0)} \\
\rho_{1}^{(1)} \varphi_{1}^{(1)}
\end{array}\right] .
\end{aligned}
$$

The aim is then to find the best beams, i.e., the amplitude and phase coefficients to represent the dominant eigenvector of the covariance matrix of the channel, which will be presented in the next section. Note that the 5G standard allows for CSI feedback for the wideband (amplitude coefficients are calculated for the entire transmission band, while the phase coefficients can be fine tuned for each subband) and subband (amplitude and phase coefficients can be fine tuned for each subband) modes. In this study, subband amplitude and phase quantization is considered to generate a CSI feedback for each subband to achieve a better performance.

\section{Quantization of the Dominant Eigenvector of the Channel}

In order to quantize the dominant eigenvector, we utilize the search method proposed in [6]. The method is based on first finding the best DFT beams and then obtaining the respective amplitude and phase combining coefficients. The steps of the method are as follows (For simplicity, we drop the user index $k$ in this subsection.)

Step 1: Determine the best beams, i.e., those with the strongest projection over the covariance matrix of the channel. 
Namely,

$$
\mathbf{b}^{*(0)}=\arg \max _{\mathbf{b}} \mathbf{b}^{H}\left(\mathbf{R}_{11}+\mathbf{R}_{22}\right) \mathbf{b},
$$

where $\mathbf{R}=\sum_{j} \mathbf{H}^{j H} \mathbf{H}^{j}=\left[\begin{array}{ll}\mathbf{R}_{11} & \mathbf{R}_{12} \\ \mathbf{R}_{21} & \mathbf{R}_{22}\end{array}\right]$ is the channel covariance matrix, with $\mathbf{H}^{j}$ being the channel matrix for the $j$-th subcarrier.

Step 2: The second beam $\mathbf{b}^{*(1)}$ is obtained using the same maximization as in (4) over the beams orthogonal to $\mathbf{b}^{*(0)}$.

Step 3: The number of possible candiates for $\mathbf{w}_{2}$ in (3) makes an exhaustive search infeasible. Hence, the quantized amplitude and phase values for the selected beams need to be obtained separately for each subband.

Step 3.1: We start by obtaining the unquantized subband amplitude coefficients. This is achieved by finding the amplitude of the dominant eigenvector of $\mathbf{W}_{1}^{* H} \mathbf{R}_{s u b} \mathbf{W}_{1}^{*}$ where $\mathbf{R}_{s u b}$ is constructed by only considering the channel matrix of the subcarriers in the subband of interest. This procedure is repeated for all the subbands.

Step 3.2: Next, we obtain the unquantized wideband amplitudes by taking the linear average of the unquantized subband amplitudes for each beam. After this, again for each beam, each subband amplitude is compared with the wideband amplitude to determine the value of the subband specific amplitude factor. The 5G-NR standard allows for 2 subband specific amplitude tuning values: $\left\{1, \frac{1}{\sqrt{2}}\right\}$.

Step 3.3: Next, the unquantized wideband amplitudes for the selected beams are quantized. In the 5G standard, the strongest beam has the quantized coefficient value of unity, and the amplitude (and the phase) of the remaining beams are determined relatively to the strongest one. The standard allows for 8 wideband quantized amplitude values $\rho=1, \frac{1}{\sqrt{2}}, \frac{1}{2}, \ldots, \frac{1}{8}, 0$. After this operation, each quantized subband amplitude can be obtained by multiplying the wideband quantized amplitude obtained in Step 3.3 and the subband specific amplitude factor obtained in Step 3.2.

Step 3.4: After finding the quantized amplitudes of each subband for each beam, the quantized phase values can be obtained by finding the closest quantized phase value to the given unquantized phase (i.e., phase of the unquantized subband coefficient). There can be $N=4$ or 8 quantized phase values according to the standard depending on the configuration, and the quantized phase values are given as $\varphi=e^{j \phi}$, where $\phi=\frac{2 \pi n}{N}$ with $n=0,1, \ldots, N-1$.

Finally, the quantized dominant eigenvector is obtained as $\mathbf{w}^{*}=\mathbf{W}_{1}^{*} \mathbf{w}_{2}^{*}[6]$.

\section{Proposed Limited Feedback Precoding Schemes}

In this section, we propose an algorithm to approximate the channel matrices using the fed-back dominant eigenvectors, which are then utilized for downlink precoding to transmit data to the users.

\section{A. Approximate Channel Reconstruction}

The BS can use the fed-back dominant eigenvector of each user as its precoding vector; however, using this method, multiuser interference cannot be suppressed well and the system performance degrades. Therefore, unless the users are physically isolated, these vectors need to be processed and updated to deal with the interference. In order to ameliorate this issue, we propose the idea of first reconstructing an equivalent channel matrix for each user employing the available fed-back eigenvector and then applying a multiuser precoding technique on the reconstructed channels for interference suppression.

We define the equivalent channel matrix of the $k$-th user, $\mathbf{H}_{k}$, as a $\mathcal{C}^{N_{r} \times N_{t}}$ matrix that satisfies $\mathbf{H}_{k}^{H} \mathbf{H}_{k}=\mathbf{R}_{k}=$ $\sum_{j} \mathbf{H}_{k, j}^{H} \mathbf{H}_{k, j}$ (with the summation over the subcarriers), where $\mathbf{R}_{k}$ is the channel covariance matrix of the $k$-th user. Using SVD, we have

$$
\mathbf{H}_{k}=\mathbf{U}_{k} \boldsymbol{\Sigma}_{k} \mathbf{V}_{k}^{H}=\sum_{i=1}^{\operatorname{rank}(\mathbf{H})} \mathbf{u}_{k, i} \sigma_{k, i} \mathbf{v}_{k, i}^{+}
$$

where $\boldsymbol{\Sigma}$ is a diagonal matrix whose diagonal elements are the singular values of $\mathbf{H}_{k}$, and $\mathbf{U}_{k}$ and $\mathbf{V}_{k}$ contain the left and right singular vectors of $\mathbf{H}_{k}$, respectively. These are equivalent to the eigenvectors of $\mathbf{R}_{k}$, with $\mathbf{u}_{k, i}$ and $\mathbf{v}_{k, i}$ being the $i$ th left and right singular vectors, respectively, and $\sigma_{k, i}$ being the $i$-th singular value of $\mathbf{H}_{k}$. The equivalent channel matrices are obtained using the known right singular vectors, and then the precoding matrices are calculated by performing an SLNRbased or LR-S-GMI-MMSE precoding algorithms.

\section{B. Single Stream: SLNR-Based Precoding}

After the approximate channel matrices are reconstructed, existing precoding algorithms for multiuser MIMO systems can be applied. Signal-to-interference-plus-noise ratio (SINR) is the most common metric in precoder design as it directly determines the capacity and link-level packet error performance of the users. However, in order to use the SINR metric, we need to jointly design the precoding vectors of the users. This often leads to non-convex optimization problems for which an analytical solution cannot be obtained. One way to decouple the precoder design problem is to use the SLNR metric proposed in [7]. The leakage from the $k$-th user to the $i$-th one is the interference power caused by the $k$-th user on the received signal of the $i$-th user, i.e., $\left\|\mathbf{H}_{i} \mathbf{p}_{k}\right\|^{2}$. Based on this, the SLNR metric of the $k$-th user is defined as the ratio of its desired signal power to the power of total noise plus the sum of leakages to the other users. For the single stream case (i.e., $r_{k}=1$ ), using (1), we have

$$
S L N R_{k}=\frac{\left\|\mathbf{H}_{k} \mathbf{p}_{k}\right\|^{2}}{N_{r} \sigma_{n}^{2}+\sum_{i=1, i \neq k}^{K}\left\|\mathbf{H}_{i} \mathbf{p}_{k}\right\|^{2}},
$$

where $\mathbf{p}_{k}$ is the precoding vector of the $k$-th user. Note that $S L N R_{k}$ is only a function of the precoding vector of the $k$-th user. The SLNR expression (6) can equivalently be written as

$$
S L N R_{k}=\frac{\left\|\mathbf{H}_{k} \mathbf{p}_{k}\right\|^{2}}{N_{r} \sigma_{n}^{2}+\left\|\tilde{\mathbf{H}}_{\mathbf{k}} \mathbf{p}_{k}\right\|^{2}},
$$


where $\tilde{\mathbf{H}}_{k}=\left[\mathbf{H}_{1}^{T} \ldots \mathbf{H}_{k-1}^{T} \ldots \mathbf{H}_{k+1}^{T} \ldots \mathbf{H}_{K}^{T}\right]^{T}$ is an extended channel matrix that only excludes $\mathbf{H}_{k}$. Maximizing (7) over $\mathbf{p}_{k}$ subject to a unit-norm constraint $\left(\left\|\mathbf{p}_{k}\right\|^{2}=1\right)$, the SLNR maximizing precoding vector of the $k$-th user is obtained as the dominant eigenvector of $\left(N_{r} \sigma_{i}^{2} \mathbf{I}+\tilde{\mathbf{H}}_{k}^{H} \tilde{\mathbf{H}}_{k}\right)^{-1} \mathbf{H}_{k}^{H} \mathbf{H}_{k}$ [8].

We have carried out simulations and found out that the SLNR-based precoding outperforms the existing algorithms in the single stream case (see Section IV). However, based on our extensive numerical results, we have observed that the performance of an alternative precoding scheme is better than that of the SLNR-based method for the full stream case $\left(r_{k}=N_{r}\right)$, which is described in the following subsection.

\section{Full Stream: LR-S-GMI}

For the full stream case (i.e., $r_{k}=N_{r}$ ), we propose to employ the LR-S-GMI algorithm with an inner MMSE precoding, called LR-S-GMI-MMSE, which has shown superior performance compared to other multiuser full stream algorithms [11]. In the following, we first describe the process of the LRS-GMI-MMSE precoding algorithm, and then we modify the receiver side to be able to utilize LR-S-GMI-MMSE for the downlink precoding with limited feedback.

Let the combined channel and precoding matrices be $\mathbf{H}=\left[\begin{array}{llll}\mathbf{H}_{1}^{T} & \mathbf{H}_{2}^{T} & \ldots & \mathbf{H}_{K}^{T}\end{array}\right]^{T} \in \mathcal{C}^{K N_{r} \times N_{t}}$ and $\mathbf{P}=$ $\left[\begin{array}{llll}\mathbf{P}_{1} & \mathbf{P}_{2} & \ldots & \mathbf{P}_{K}\end{array}\right] \in \mathcal{C}^{N_{t} \times K N_{r}}$, respectively. In the LR-SGMI-MMSE algorithm, the precoding matrix is obtained in two steps as in the BD-type precoding algorithms. Therefore, the precoding matrix of $k$-th user can be expressed as $\mathbf{P}_{k}=\mathbf{P}_{k}^{a} \mathbf{P}_{k}^{b}$, where $\mathbf{P}_{k}^{a} \in \mathcal{C}^{N_{t} \times N_{t}}$ and $\mathbf{P}_{k}^{b} \in \mathcal{C}^{N_{t} \times N_{r}}$.

At the first step, $\mathbf{P}_{k}^{a}$ is obtained by a channel inversion and $K \mathrm{QR}$ decompositions as follows. First, an MMSE channel inversion is applied to the combined channel matrix, i.e.,

$$
\begin{aligned}
\mathbf{H}_{m s e}^{+} & =\mathbf{H}^{H}\left(\mathbf{H H}^{H}+\alpha \mathbf{I}\right)^{-1} \\
& =\left[\begin{array}{lll}
\mathbf{H}_{1, m s e}, & \ldots & \mathbf{H}_{K, m s e}
\end{array}\right],
\end{aligned}
$$

where $\mathbf{H}_{k, m s e} \in \mathcal{C}^{N_{t} \times N_{r}}$ is the sub-matrix of $\mathbf{H}_{m s e}^{+}$and $\alpha=\frac{K N_{r} \sigma_{n}^{2}}{\xi}$ is the regularization factor with $\xi$ being the average transmit power. The first precoding matrix of the $k$ th user is then $\mathbf{P}_{k}^{a}=\mathbf{Q}_{k, m s e}$ where $\mathbf{Q}_{k, m s e}$ is obtained by the QR decomposition of $\mathbf{H}_{k, m s e}$, i.e., $\mathbf{H}_{k, m s e}=\mathbf{Q}_{k, m s e} \mathbf{R}_{k, m s e}$. Hence, the first combined precoding matrix can be expressed as $\mathbf{P}^{a}=\left[\mathbf{P}_{1}^{a}, \mathbf{P}_{2}^{a}, \ldots \mathbf{P}_{K}^{a}\right]$.

In the second step, the MU-MIMO channel can be transformed into parallel or approximately parallel single user MIMO (SU-MIMO) channels as follows

$$
\mathbf{H}_{e f f_{k}}=\mathbf{H}_{k} \mathbf{P}_{k}^{a},
$$

where $\mathbf{H}_{e f f_{k}}$ is the effective channel matrix of the $k$-th user.

Lenstra-Lenstra-Lovász (LLL) lattice basis reduction algorithm is the most common LR method, which provides a short, nearly orthogonal basis in polynomial time. Before obtaining the second precoding matrix $\left(\mathbf{P}_{k}^{b}\right)$, a complex version of LLL algorithm [14] is applied to the effective channel matrix to obtain the LLL-transformed effective channel matrix as follows

$$
\underline{\tilde{\mathbf{H}}}_{e f f_{k}}=\underline{\mathbf{T}}_{k} \underline{\mathbf{H}}_{e f f_{k}},
$$

where $\underline{\mathbf{H}}_{e f f_{i}}^{T}=\left[\mathbf{H}_{e f f_{i}}, \sqrt{\alpha} \mathbf{I}_{N_{i}}\right]^{T}$ and $\underline{\mathbf{T}}_{k}$ is the LLL transform matrix, which is a unimodular matrix with complex integer entries and $\operatorname{det}\left(\underline{\mathbf{T}}_{k}\right)=1$. Employing the inner MMSE precoding, the second precoding matrix of the $k$-th user is calculated as

$$
\tilde{\mathbf{P}}_{k}^{b}=\mathbf{A}_{k} \underline{\tilde{\mathbf{H}}}_{e f f_{k}}^{H}\left(\underline{\tilde{\mathbf{H}}}_{e f f_{k}} \underline{\tilde{\mathbf{H}}}_{e f f_{k}}^{H}\right)^{-1},
$$

where $\mathbf{A}_{k}=\left[\begin{array}{ll}\mathbf{I}_{M} & \mathbf{0}_{M \times N_{r}}\end{array}\right]$. The combined second precoding matrix $\mathbf{P}^{b}$ is

$$
\mathbf{P}^{b}=\operatorname{diag}\left\{\tilde{\mathbf{P}}_{1}^{b}, \tilde{\mathbf{P}}_{2}^{b}, \ldots, \tilde{\mathbf{P}}_{K}^{b}\right\},
$$

where $\operatorname{diag}\left\{\tilde{\mathbf{P}}_{\tilde{\mathbf{P}}}^{b}, \tilde{\mathbf{P}}_{2}^{b}, \ldots, \tilde{\mathbf{P}}_{K}^{b}\right\}$ is a block diagonal matrix with the matrices $\tilde{\mathbf{P}}_{k}^{b}$ on its main diagonal. The overall precoding matrix is obtained as $\mathbf{P}=\mathbf{P}^{a} \mathbf{P}^{b}$.

Based on (1), the received signal of the $k$-th user $\mathbf{y}_{k}$ consists of the desired signal $\mathbf{H}_{k} \mathbf{P}_{k} \mathbf{s}_{k}$ and the multiuser interference plus noise. If there is perfect CSI at the transmitter $\mathbf{H}_{k} \mathbf{P}_{k}$ reduces to $\underline{\mathbf{T}}_{k}^{-1}$. Therefore, possible desired signal points can be obtained by $\underline{\mathbf{T}}_{k}^{-1} \mathbf{s}_{k}$. In order to perform a maximum likelihood (ML) type detection, the received signal $\mathbf{y}_{k}$ can be quantized to the nearest possible signal point $\tilde{\mathbf{s}}_{k}$. Since the receiver has the CSI knowledge (hence it can calculate $\underline{\mathbf{T}}_{k}$ ), the final estimated symbols can be obtained by inverting the LLL transform, i.e., through $\hat{\mathbf{s}}_{k}=\underline{\mathbf{T}}_{k} \tilde{\mathbf{s}}_{k}$.

Since the lattice reduced matrix $\mathbf{P}^{b}$ has near orthogonal columns, the required transmit power is reduced and a better BER performance can be obtained compared to other BD-type algorithms such as the regularized BD (RBD) [11]. This has motivated us to employ the LR-S-GMI-MMSE algorithm in the limited feedback setup. However, the perfect CSI is not known at the BS for our case, hence we need to modify the receiver side to be able to employ the LR-S-GMI-MMSE algorithm. To accomplish this, we replace the combined channel matrix $\mathbf{H}$ with the estimated reconstructed channel (see Section III.A). Then, we apply the LR-S-GMI-MMSE algorithm to the reconstructed channel and obtain the LR-aided precoding matrix. Clearly, since the precoding matrix is obtained using only an approximate channel knowledge, $\mathbf{H}_{k} \mathbf{P}_{k}$ does not reduce to $\underline{\mathbf{T}}_{k}^{-1}$ as in the perfect CSI case. Hence, in order to apply an MLtype detection, we propose that after quantizing the received signal $\mathbf{y}_{k}$ to the nearest possible signal points $\tilde{\mathbf{s}}_{k}$, the final estimated symbols $\left(\hat{\mathbf{s}}_{k}\right)$ are obtained as $\hat{\mathbf{s}}_{k}=\left(\mathbf{H}_{k} \mathbf{P}_{k}\right)^{-1} \tilde{\mathbf{s}}_{k}$. A pseudo-code of the proposed precoding scheme is given in Algorithm 1.

\section{Complexity Analysis}

A complexity analysis for the proposed and reference precoding methods at the BS is presented in this subsection. Since the feedback part is common for both our proposed method and the methods we use for comparison, which are SVD-SI [12] and S-MMSE based method proposed in [13], the main comparison is on the applied precoding methods at the BS. In what follows, we present a per layer complexity analysis for 


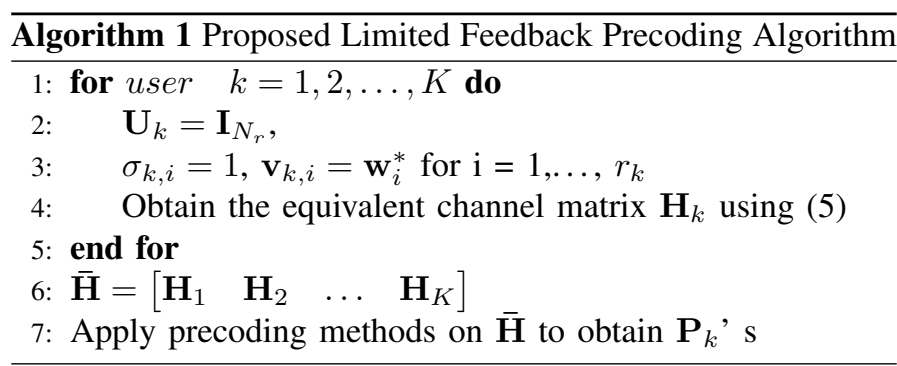

all these methods.

For our proposed method, the main operations are channel reconstruction and SLNR precoding. The channel reconstruction is only a matrix multiplication with complexity of $\mathcal{O}\left(N_{r} N_{t}\right)$. Complexity of the SLNR precoding is given as $\mathcal{O}\left(N_{t}^{3}\right)$ in [7], which dominates the complexity of our method. Since there are $K$ users, overall complexity of our algorithm with a straightforward implementation is $\mathcal{O}\left(K N_{t}^{3}\right)$.

For the S-MMSE based method in [13], the precoder of each user is determined by Eq. (6), where the dominant part in complexity is calculation of the inverse of an $N_{t} \times N_{t}$ matrix, which is $\mathcal{O}\left(N_{t}^{3}\right)$. Therefore, the overall complexity of the SMMSE based method is also $\mathcal{O}\left(K N_{t}^{3}\right)$. For the SVD-SI, all users' precoders are obtained as described in [12], where a complexity of $\mathcal{O}\left(K^{2} N_{t}\right)$ is required for the matrix multiplication and $\mathcal{O}\left(K^{3}\right)$ for the matrix inversion. Since $K<N_{t}$, the complexity of SVD-SI is $\mathcal{O}\left(K^{2} N_{t}\right)$. Therefore, it can be concluded that the complexity of the proposed algorithm and the S-MMSE based method in [13] are similar, while that of the SVD-SI based method is lower.

\section{Numerical Results}

We now compare the performance of the proposed algorithm with those of the SVD-SI algorithm in [12] and the algorithm proposed in [13] based on the S-MMSE method. We assume that the transmitted symbols are chosen randomly and endently from a quadrature phase-shift keying (QPSK) constellation and that the users perform an MMSE equalization. The simulation parameters are chosen according to the 5G-NR standard. We consider a single cell network with $K=4$ users (each equipped with $N_{r}=2$ antennas). We consider the cluster delay line-B (CDL-B) [15] as the channel model and cyclicprefix orthogonal frequency division multiplexing (CP-OFDM) with $30 \mathrm{kHz}$ subcarrier spacing as the waveform. We also assume that the users transmit the dominant eigenvectors of the covariance matrix of each subband by using a DFT Type2 codebook of 5G-NR with parameters $\left(N_{1}, N_{2}, O_{1}, O_{2}\right)$ [15] with $L=3$, where $N_{1}$ and $N_{2}$ are the numbers of cross polar antenna columns and rows of the $2 \mathrm{D}$ antenna array of the BS, respectively, and $O_{1}$ and $O_{2}$ are the oversampling factors of the DFT codebook for each dimension. Note that, considering polarization, the number of antenna ports at the BS is $N_{t}=2 N_{1} N_{2}$. Channel coding is employed using a rate $3 / 4$ low-density parity-check (LDPC) code of blocklength 4800 for the single stream case, and an LDPC code of length 9152

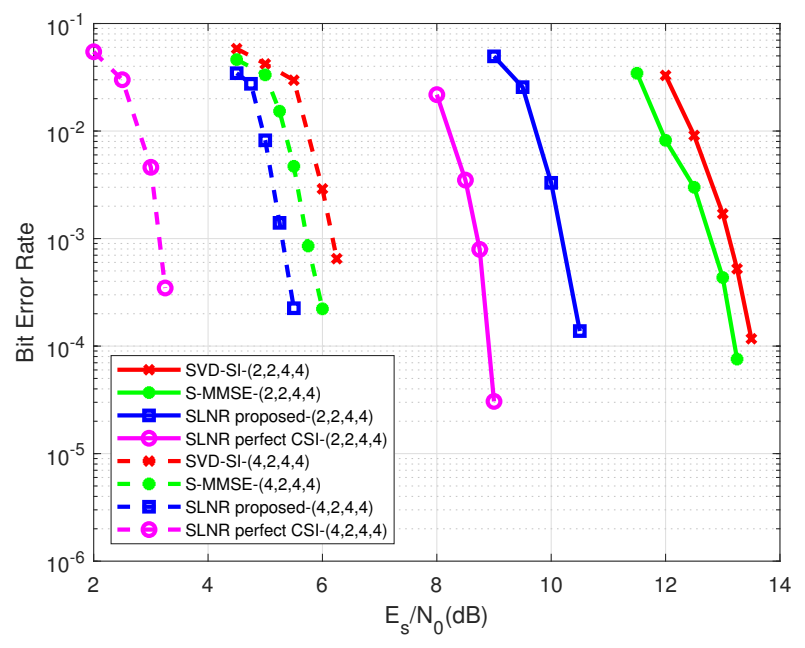

Fig. 1. BER results of the single stream case for a delay spread of $30 \mathrm{~ns}$.

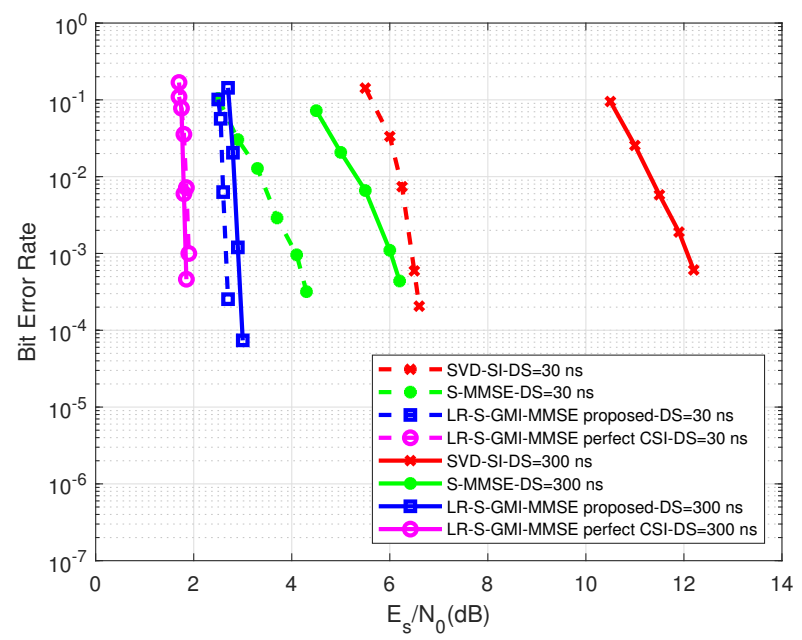

Fig. 2. BER results for the full stream case with $(4,2,4,4)$ DFT Type-2 codebook.

and rate 0.1923 taken from the 5G-NR standard [16] for the full stream case.

The bit error rate (BER) results of the single stream case for the $(2,2,4,4)$ and $(4,2,4,4)$ DFT Type-2 codebook and the CDL-B channel for a delay spread (DS) value of $30 \mathrm{~ns}$ are presented in Fig. 1. We have also provided the perfect CSI results as a benchmark, in which it is assumed that the BS has the perfect knowledge of the users' channel matrices. As can be seen from Fig. 1, the proposed algorithm outperforms the S-MMSE based method in [13] by $2.5 \mathrm{~dB}$ and SVD-SI in [12] by about $3 \mathrm{~dB}$ at a bit error rate of $10^{-3}$ in the $(2,2,4,4)$ DFT Type- 2 configuration. In the $(4,2,4,4)$ configuration, the proposed algorithm is superior to the S-MMSE based method by $0.4 \mathrm{~dB}$ and to the SVD-SI by $0.8 \mathrm{~dB}$. In addition, increasing the number of BS antennas from 8 to 16 considerably improves the BER performance.

We also present the BER results for the full stream case with 


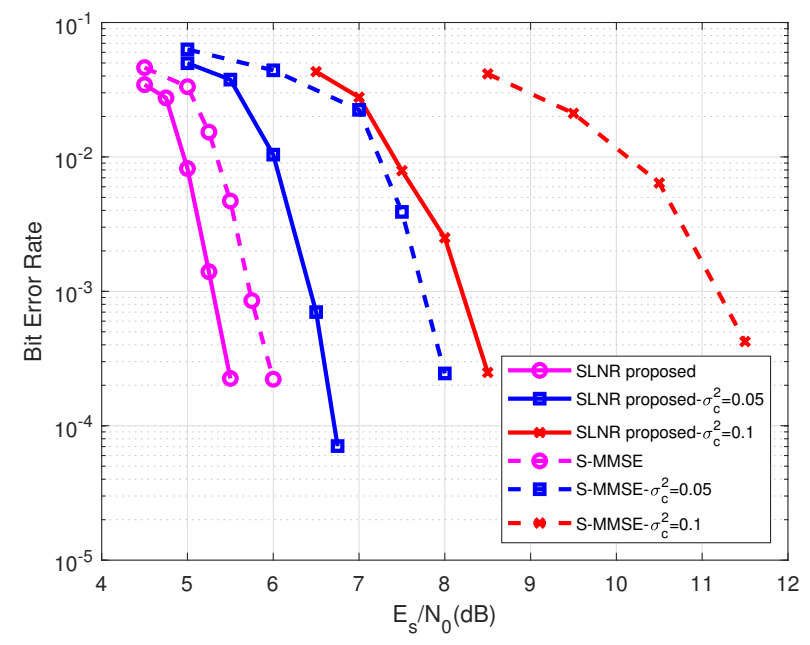

Fig. 3. BER results for the case of channel estimation errors with $(4,2,4,4)$ DFT Type- 2 codebook and delay spread of $30 \mathrm{~ns}$

the $(4,2,4,4)$ DFT Type-2 codebook in Fig. 2. The proposed algorithm outperforms the S-MMSE based method by about $1.5 \mathrm{~dB}$ and $3.5 \mathrm{~dB}$, and the SVD-SI based method about 3.5 $\mathrm{dB}$ and $8.5 \mathrm{~dB}$ for the delay spread values of $30 \mathrm{~ns}$ and 300 ns, respectively. Comparing the results in Fig. 1 and Fig. 2, we observe that while the data rate increases by increasing the number of data streams (from single stream to full stream), the BER performance worsens for all algorithms (i.e., there is a rate-error tradeoff). Also, the performance degrades with increasing the delay spread as expected.

In Fig. 1 and Fig. 2, we assume that the users have perfect knowledge of their downlink channels. However, in practice, channel estimation errors occur, which degrades the system performance. We also consider this scenario by modelling the channel estimates at the users as $\mathbf{H}_{i}^{\prime}=\mathbf{H}_{i}+\boldsymbol{\Gamma}_{i}$, where $\boldsymbol{\Gamma}_{i}$ denotes the error matrix with i.i.d. zero mean complex Gaussian elements with variance $\sigma_{c}^{2}$. We use $\mathbf{H}_{i}^{\prime}$ instead of $\mathbf{H}_{i}$ to obtain the feedback, and keep the rest of the precoding procedure the same. We consider a case with DS of $30 \mathrm{~ns}$, and DFT Type2 configuration with $(4,2,4,4)$. Fig. 3 demonstrates that the proposed algorithm is more robust to the channel estimation errors as the performance gain with respect to the S-MMSE based method (we use it for comparison as the performance of the SVD-SI method is worse) is $0.4 \mathrm{~dB}$ for the case of no estimation errors, $1.25 \mathrm{~dB}$ for $\sigma_{c}^{2}=0.05$ and $3 \mathrm{~dB}$ for $\sigma_{c}^{2}=0.1$. A possible reason of this observation could be that the increased quantization error in the fed-back dominant eigenvector due to the channel estimation errors degrades the performance of the S-MMSE based method more since it directly depends on the fed-back dominant eigenvector.

\section{CONCLUSION}

We have considered the problem of multiuser precoding with limited feedback, assuming that the BS has access to only the dominant eigenvector of the covariance matrix of each user's channel. We have proposed an algorithm which first reconstructs the approximate channel matrices using the limited feedback, and then, performs downlink precoding. An SLNR maximization based precoding is employed for the single stream case, while a modified version of the LR-S-GMI-MMSE algorithm is used for the full stream case. For analysis, we have considered a realistic setup with DFT Type-2 structure and CDL-B channel model defined in the 5G-NR standard. Extensive simulation results illustrate the superiority of the proposed algorithm over the existing eigenvector based precoders such as the SVD-SI and the S-MMSE-based method in both single stream and full stream scenarios, and its robustness to the downlink channel estimation errors.

\section{REFERENCES}

[1] D. J. Love, R. W. Heath, V. K. N. Lau, D. Gesbert, B. D. Rao and M. Andrews, "An overview of limited feedback in wireless communication systems," IEEE J. Sel. Areas Commun., vol. 26, no. 8, pp. 1341-1365, Oct. 2008.

[2] J. Park, J. Um, Y. Park and B. J. Jeong, "GSVD based multi-user multistream MIMO linear precoding with limited feedback," in Proc. IEEE 23rd International Symposium on Personal, Indoor and Mobile Radio Communications (PIMRC), Sydney, NSW, 2012, pp. 1874-1879.

[3] J. Mirza, P. A. Dmochowski, P. J. Smith and M. Shafi, "A differential codebook with adaptive scaling for limited feedback MU MISO systems," IEEE Wireless Commun. Lett., vol. 3, no. 1, pp. 2-5, Feb. 2014.

[4] H. Shen, W. Xu, S. Jin and C. Zhao, "Joint transmit and receive beamforming for multiuser MIMO downlinks with channel uncertainty," IEEE Trans. Veh. Tech., vol. 63, no. 5, pp. 2319-2335, Jun. 2014.

[5] J. Mirza, P. J. Smith, M. Shafi, P. A. Dmochowski, A. Firag and A. Papathanassiou, "Double-cap differential codebook structure for MU MISO systems in correlated channels," IEEE Wireless Commun. Lett., vol. 3, no. 4, pp. 441-444, Aug. 2014.

[6] B. Mondal, V. Sergeev, A. Sengupta and A. Davydov, "5G-NR (New Radio) CSI computation algorithm and performance," in Proc. Asilomar Conference on Signals, Systems, and Computers, Pacific Grove, CA, USA, 2018, pp. 1068-1071.

[7] M. Sadek, A. Tarighat and A. H. Sayed, "A leakage-based precoding scheme for downlink multi-user MIMO channels," IEEE Trans. Wireless Commun., vol. 6, no. 5, pp. 1711-1721, May 2007.

[8] M. Sadek, A. Tarighat, and A. H. Sayed, "Active antenna selection in multiuser MIMO communications," IEEE Trans. Signal Process., vol. 55, no. 4, pp. 1498-1510, April 2007.

[9] M. R. Bremner, Lattice Basis Reduction: An Introduction to the LLL Algorithm and its Applications. CRC Press, 2011.

[10] H. Sung, S. Lee and 1. Lee, "Generalized channel inversion methods for multiuser MIMO systems," IEEE Trans. Commun., vol. 57, no. 2, pp. 34893409, Nov. 2009.

[11] K. Zu, R. C. de Lamare and M. Haardt, "Generalized design of lowcomplexity block diagonalization type precoding algorithms for multiuser MIMO systems," IEEE Trans. Commun., vol. 61, no. 10, pp. 4232-4242, Oct. 2013.

[12] Y. Yang, L. Tian, J. Zhou, Y. Sun, J. Shi and Z. Li, "An efficient transmission scheme with limited feedback in multiuser MIMO systems," in Proc. IEEE Int. Conf. on Commun., Beijing, 2008, pp. 3367-3371.

[13] B. Mondal et al., "MU-MIMO and CSI feedback performance of NR/LTE," in Proc. Annual Conference on Information Sciences and Systems (CISS), Baltimore, MD, USA, 2019, pp. 1-6

[14] Y. H. Gan, C. Ling and W. H. Mow, "Complex lattice reduction algorithm for low-complexity full-diversity MIMO detection," IEEE Trans. Signal Process., vol. 57, no. 7, pp. 2701-2710, July 2009.

[15] 3GPP TR 38.901, Study on channel model for frequencies from 0.5 to 100 GHz. Rel. 14.1.1, July 2017.

[16] 3GPP TS 38.212, 5G-NR: Multiplexing and channel coding. Rel. 15.2.0, July 2018. 